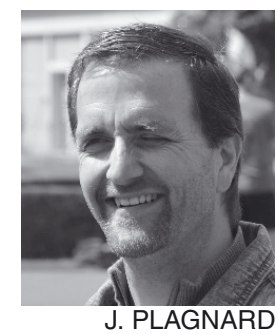

\title{
Mesure de spectres en énergie de l'émission de tubes à rayons $X$ au LNE-LNHB/LMD
}

\section{Measurement of the LNE-LNHB/LMD X-ray tube spectra by $X$-ray spectrometry}

\author{
Johann PLAGNARD \\ CEA, LIST, Laboratoire national Henri Becquerel (LNE-LNHB), Bât 602 PC111, CEA-Saclay 91191 Gif-sur-Yvette Cedex, France, \\ johann.plagnard@cea.fr.
}

\begin{abstract}
Résumé
Durant ces quatre dernières années, le LNE-LNHB/LMD s'est attaché à développer des méthodes et des moyens pour mesurer la répartition énergétique de l'émission $\mathrm{X}$ des tubes à rayons $\mathrm{X}$ utilisés pour la réalisation des références en kerma dans l'air et leurs transferts vers des laboratoires utilisateurs pour des besoins médicaux ou industriels. Deux bancs de mesure intégrant des détecteurs à semi-conducteurs (SiPIN, GeHP, CdTe) équipés de collimateurs comportant de très faibles diamètres d'ouverture, ainsi que des plateformes de positionnement ont été réalisés. Ces plateformes assurent un alignement optimal du couple détecteur/collimateur sur l'axe des faisceaux. Des méthodes de correction des artéfacts de détection ont été développées pour chaque type de détecteur et intégrées dans des programmes de correction des spectres mesurés. Une campagne de caractérisation de 28 faisceaux de référence du LNE-LNHB/LMD, a été menée et a permis de tester et de valider l'ensemble des algorithmes de correction de spectres développés au laboratoire. Les résultats obtenus ont été comparés aux spectres d'émission calculés à l'aide de logiciels commercialisés.
\end{abstract}

MOTS CLÉS : TUBE À RAYONS X, SPECTRE D'ÉNERGIE, DÉTECTEUR GEHP, DÉTECTEUR CDTE, DÉTECTEUR SI-PIN, DOSIMÉTRIE X.

\footnotetext{
Abstract

For the last four years, the LNE-LNHB/LMD has been developing methods and material to measure the X-ray spectra of its X-ray tubes used to perform primary standards and transfers in medical or industrial fields. Two different measuring devices have been built. They include the possibility to use three different semiconductor detectors (Si-PIN, GeHP, CdTe) equipped with tungsten collimators with small apertures. Two rotation and translation stages were included to these benches for an automatic and precise alignment of the couple detector/collimator on the beam axis. Correction methods were developed for each detector, to take into account all the detection artefacts taking place into the semi-conductor crystal. They were included in specific spectrum correction programs. Characterization
}

of 28 LNE-LNHB/LMD reference beams was carried out. It allows testing and validating all the different algorithms of spectrum correction developed at the laboratory. These results were compared to the calculated spectra obtained with the XCOMP5r and SpekCalc V1.0 software.

KEY WORDS: X-RAY TUBE, SPECTRUM, GEHP DETECTOR, CDTE DETECTOR, SI-PIN DETECTOR, X-RAY DOSIMETRY.

\section{Introduction}

Dans le domaine de la dosimétrie des rayonnements photoniques d'énergie inférieure à $300 \mathrm{keV}$, la connaissance de la distribution énergétique des photons émis par les générateurs de rayons $\mathrm{X}$ constitue un paramètre important. En effet, dans le cadre de la chaîne métrologique existant entre les laboratoires nationaux de métrologie, les laboratoires d'étalonnage accrédités et les utilisateurs, il est indispensable de s'assurer que les faisceaux utilisés par chaque laboratoire sont rigoureusement similaires. Pour ce faire, des normes internationales ont été établies pour définir les caractéristiques des faisceaux spécifiques répondant aux besoins des utilisateurs (exemple : norme ISO 4037-1 relative aux rayonnements $X$ et gamma de référence pour l'étalonnage des dosimètres et des débitmètres [1]). Des indices de qualité des faisceaux ont ainsi été définis et correspondent, pour la plupart, à la mesure des couches de demi-atténuation (CDA) des faisceaux. Or, plus les énergies des faisceaux sont faibles (inférieures à $50 \mathrm{keV}$ ), plus il est complexe de réaliser des faisceaux de référence, ceci à cause des phénomènes importants d'absorption qui déforment les spectres d'émission photoniques dans cette gamme d'énergie. En pratique, 
pour définir la qualité d'un faisceau, la mesure des CDA s'avère être un indicateur limité et non univoque. En effet, pour une valeur de CDA donnée, plusieurs faisceaux peuvent lui correspondre.

C'est pourquoi le LNE-LNHB s'est attaché, depuis plusieurs années, à développer une méthode de mesure directe des spectres d'énergie du rayonnement émis par les tubes à rayons $\mathrm{X}$, à l'aide de détecteurs à semiconducteurs. Trois types de détecteurs ont été utilisés afin de répondre aux différentes gammes d'énergies couvertes par les faisceaux de référence du LNE-LNHB (10 keV à $300 \mathrm{keV}$ ) : un détecteur au silicium du type Si-PIN, un détecteur au germanium hyper-pur GeHP et enfin un détecteur au CdTe. Ces détecteurs ont été équipés de collimateurs en tungstène de très faible ouverture afin de limiter le flux très important émis par les tubes à rayons X. Du fait des contraintes d'alignement occasionnées par l'utilisation de ces petits collimateurs, des bancs motorisés ont été développés, permettant de positionner précisément les détecteurs sur l'axe du faisceau.

Malgré cette importante collimation, les déformations liées aux empilements électroniques restent toujours visibles sur les spectres mesurés. Un logiciel de traitement des spectres a donc été développé afin de soustraire les effets des empilements résiduels sur les spectres. Deux autres programmes de correction adaptés aux détecteurs CdTe et GeHP ont été développés afin de corriger des spectres mesurés les déformations spectrales dues aux défauts de détection (photons diffusés, échappements de photons de fluorescence, défauts de collection des charges électriques, rendements de détection). La réalisation de ces programmes a nécessité la caractérisation des systèmes de détection (détecteurs et électronique associée), à l'aide d'une source de rayonnement monochromatique, permettant de connaître les réponses spectrales des détecteurs utilisés dans la gamme d'énergies comprises entre $26 \mathrm{keV}$ et $250 \mathrm{keV}$. L'installation utilisée correspond à la ligne de lumière «ID17 » de l'ESRF (European Synchrotron Radiation Facility) à Grenoble.

À l'issue de ce travail de caractérisation et de développement d'algorithmes de correction, une campagne de mesures des spectres en énergie de 28 faisceaux de rayonnement X de référence du LNE-LNHB a été réalisée. Ces spectres ont ensuite été comparés aux spectres calculés avec deux logiciels commercialisés «XCOMP5r» [2] et «SpekCalc V1.0» [3].

\section{Faisceaux $X$ de référence du LNE-LNHB}

Pour réaliser cette campagne de mesure des spectres énergétiques des émissions $\mathrm{X}$, nous avons choisi trois installations parmi l'ensemble du parc de matériel utilisé en dosimétrie des rayons X au LNE-LNHB. Il s'agit d'un mammographe de marque General Electric délivrant un faisceau pulsé et de deux tubes à rayons $\mathrm{X}$ (modèles « Gulmay $160 \mathrm{kV}$ » et « Seifert $320 \mathrm{kV}$ ») qui délivrent des faisceaux continus.
Tableau 1

Caractéristiques du détecteur Si-PIN.

\begin{tabular}{|l|l|}
\hline Technologie & $\begin{array}{l}\text { Silicium refroidi par double } \\
\text { étage Peltier }\end{array}$ \\
\hline Marque & Amptek \\
\hline Type de détecteur & XR-100CR \\
\hline Surface du cristal & $13 \mathrm{~mm}^{2}$ \\
\hline Epaisseur du cristal & $50 \mu \mathrm{m}$ \\
\hline Collimateur interne & $\mathrm{W}: 10 \mu \mathrm{m}, \mathrm{Cr}: 35 \mu \mathrm{m}$, \\
multicouches & $\mathrm{Ti}: 15 \mu \mathrm{m}, \mathrm{Al}: 75 \mu \mathrm{m}$ \\
\hline Fenêtre d'entrée & $\mathrm{Be}: 25 \mu \mathrm{m}$ \\
\hline Résolution à 5,9 keV & $198 \mathrm{eV}$ \\
\hline
\end{tabular}

Les faisceaux générés par le mammographe sont obtenus à partir d'une anode et d'une filtration interne en Molybdène (référence : Mo-Mo). Différentes hautes tensions ont été utilisées : $22 \mathrm{kV}, 25 \mathrm{kV}, 28 \mathrm{kV}, 30 \mathrm{kV}$ et $35 \mathrm{kV}$.

Concernant le tube $\mathrm{X}$ « Gulmay $160 \mathrm{kV}$ », les faisceaux générés ont les désignations suivantes : CCRI10, CCRI25, CCRI30, CCRI 50a, CCRI50b, N15, N20, N30, $\mathrm{N} 40$ et N60. Ils répondent aux caractéristiques décrites dans la norme ISO 4037-1.

Les faisceaux du tube «Seifert $320 \mathrm{kV}$ » répondent aux normes ISO 4037-1 et NE EN 61267; ils sont référencés : CCRI100, CCRI135, RQR6, RQR9, RQR10, N20, N30, N40, N60, N80, N100, N120 et N150.

\section{Détecteurs, collimateur et bancs de mesure}

\subsection{Détecteur Si-PIN}

Le détecteur Si-PIN utilisé au LNE-LNHB pour la caractérisation des faisceaux de rayons $\mathrm{X}$ correspond à un détecteur au silicium refroidi par un module à effet Peltier à double étage. L'adoption de ce système de refroidissement permet d'avoir un encombrement très réduit ainsi qu'un temps de mise en œuvre très rapide. De plus, l'abaissement de sa température de fonctionnement à $-58{ }^{\circ} \mathrm{C}$ permet de diminuer le bruit électronique du détecteur, ce qui améliore sa résolution spectrale. Les principales caractéristiques de ce détecteur sont présentées dans le tableau 1.

L'épaisseur active de $500 \mu \mathrm{m}$ de ce détecteur est relativement faible. La masse volumique du silicium n'est pas très élevée : $2,34 \mathrm{~g} \cdot \mathrm{cm}^{-3}$. L'effet combiné d'une épaisseur faible et de la densité peu importante du silicium ne permet pas l'emploi de ce type de détecteur pour la mesure des énergies supérieures à quelques dizaines de kiloélectronvolts. Nous avons donc limité l'utilisation du détecteur Si-PIN à la mesure des énergies inférieures à $30 \mathrm{keV}$.

\subsection{Détecteur CdTe}

Le détecteur CdTe utilisé au LNE-LNHB pour la caractérisation des faisceaux de rayons $\mathrm{X}$ correspond à un détecteur au tellure de cadmium refroidi par un module à effet Peltier (fig. 1). Les caractéristiques fournies 


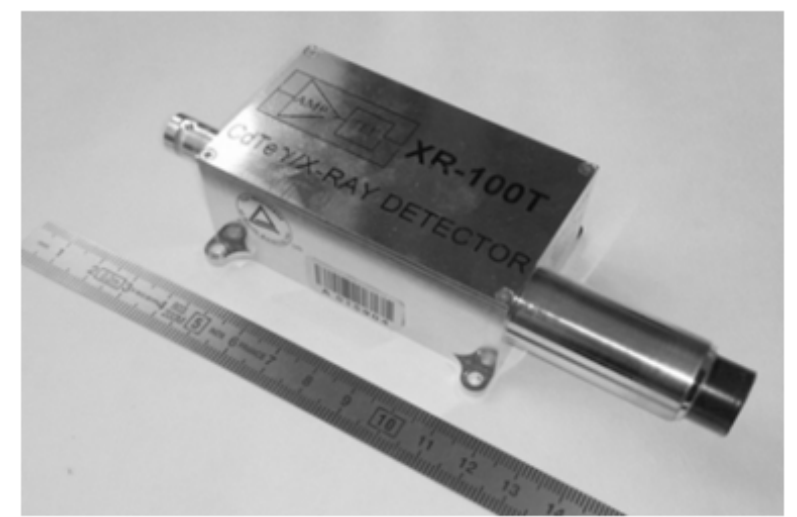

Fig. 1. - Détecteur CdTe refroidi par effet Peltier.

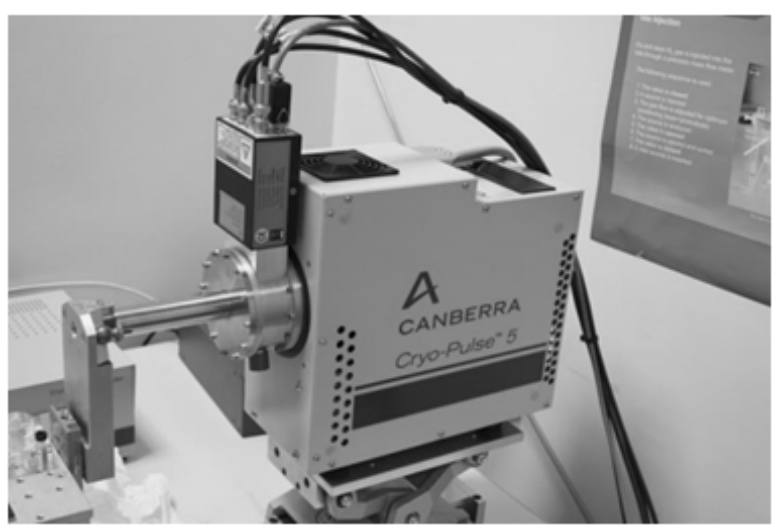

Fig. 2. - Détecteur GeHP refroidi par cryogénérateur.

par le constructeur sont présentées dans le tableau 2. Ce détecteur est équipé d'un préamplificateur capable de mesurer des taux de comptage élevés tout en minimisant les déformations liées à la saturation du préamplificateur. La masse volumique importante du cristal de CdTe $\left(5,85 \mathrm{~g} \cdot \mathrm{cm}^{-3}\right)$ et son épaisseur de $1 \mathrm{~mm}$ permettent de détecter des photons de plusieurs centaines de kiloélectronvolts.

\subsection{Détecteur GeHP}

Le détecteur GeHP utilisé au LNE-LNHB pour la caractérisation des faisceaux de rayons $\mathrm{X}$ correspond à un détecteur au germanium hyper-pur (GeHP), refroidi par un cryogénérateur électrique (fig. 2). Les caractéristiques fournies par le constructeur sont présentées dans le tableau 3.

Ce détecteur est également équipé d'un préamplificateur permettant la détection des forts taux de comptage. Il est doté d'un cryogénérateur permettant d'abaisser sa température de fonctionnement à $-150{ }^{\circ} \mathrm{C}$. Malgré une taille réduite par rapport à un cryostat classique utilisant de l'azote liquide, ce système est assez lourd et l'ensemble pèse près de $18 \mathrm{~kg}$. Le positionnement très précis d'une telle masse dans l'axe d'un faisceau a nécessité le développement d'un banc de déplacement équipé de platines de translation et de rotation volumineuses et lourdes. La masse volumique de $5,32 \mathrm{~g} \cdot \mathrm{cm}^{-3} \mathrm{du}$ germanium ainsi que l'épaisseur importante du cristal $(10 \mathrm{~mm})$ offrent une
Tableau 2

Caractéristiques du détecteur CdTe.

\begin{tabular}{|l|l|}
\hline Technologie & $\begin{array}{l}\text { Tellure de cadmium refroidi } \\
\text { par étage Peltier }\end{array}$ \\
\hline Marque & Amptek \\
\hline Type de détecteur & XR-100T \\
\hline Surface du cristal & $9 \mathrm{~mm}^{2}$ \\
\hline Épaisseur du cristal & $1 \mathrm{~mm}$ \\
\hline Fenêtre d'entrée & $\mathrm{Be}: 100 \mu \mathrm{m}$ \\
\hline Résolution à $59,5 \mathrm{keV}$ & $560 \mathrm{eV}$ \\
\hline
\end{tabular}

Tableau 3

Caractéristiques du détecteur GeHP.

\begin{tabular}{|l|l|}
\hline Technologie & $\begin{array}{l}\text { Germanium hyper pur refroidi } \\
\text { par cryogénérateur électrique }\end{array}$ \\
\hline Marque & Canberra \\
\hline Type de détecteur & GUL0110 \\
\hline Type de cryostat & Cryogénérateur model CP-5F \\
\hline Surface du cristal & $100 \mathrm{~mm}^{2}$ \\
\hline Epaisseur du cristal & $10 \mathrm{~mm}$ \\
\hline Fenêtre d'entrée & $\mathrm{Be}: 25 \mu \mathrm{m}$ \\
\hline Résolution à $59,5 \mathrm{keV}$ & $355 \mathrm{eV}$ \\
\hline
\end{tabular}

dynamique de mesure suffisante, jusqu'à plusieurs centaines de kiloélectronvolts.

\subsection{Système de collimation}

Afin de réduire le taux de comptage incident sur chaque détecteur, un ensemble de collimateurs en tungstène a été systématiquement positionné sur leur face d'entrée. Les différentes configurations adoptées dépendent des tubes à rayons X à mesurer :

- Mammographe : 1 collimateur de $50 \mu \mathrm{m}$ d'ouverture et de $2 \mathrm{~mm}$ d'épaisseur;

- Gulmay 160 kV : 8 collimateurs de $500 \mu \mathrm{m}$ d'ouverture et d'épaisseur $2 \mathrm{~mm}+4$ collimateurs de $150 \mu \mathrm{m}$ d'ouverture et d'épaisseur $2 \mathrm{~mm}$;

- Siefert $320 \mathrm{kV}$ : 9 collimateurs de $500 \mu \mathrm{m}$ d'ouverture et d'épaisseur $2 \mathrm{~mm}+5$ collimateurs de $150 \mu \mathrm{m}$ d'ouverture et de $2 \mathrm{~mm}$ d'épaisseur.

Pour les spectres de «fortes énergies » mesurés sur le tube X Gulmay $320 \mathrm{kV}$ et pour éviter la détection de photons diffusants sur les structures des détecteurs, une plaque de blindage en plomb, d'épaisseur $1 \mathrm{~cm}$ et équipée d'un trou de passage de diamètre $1 \mathrm{~cm}$, a été placée entre le système de collimation et la sortie du tube à rayons $\mathrm{X}$.

\subsection{Bancs de positionnement et distances de mesure}

L'utilisation de ces petits collimateurs rend les opérations d'alignement des détecteurs sur l'axe du faisceau très délicates. Afin de pallier cette difficulté, deux bancs de positionnement ont été développés. Le premier offre 


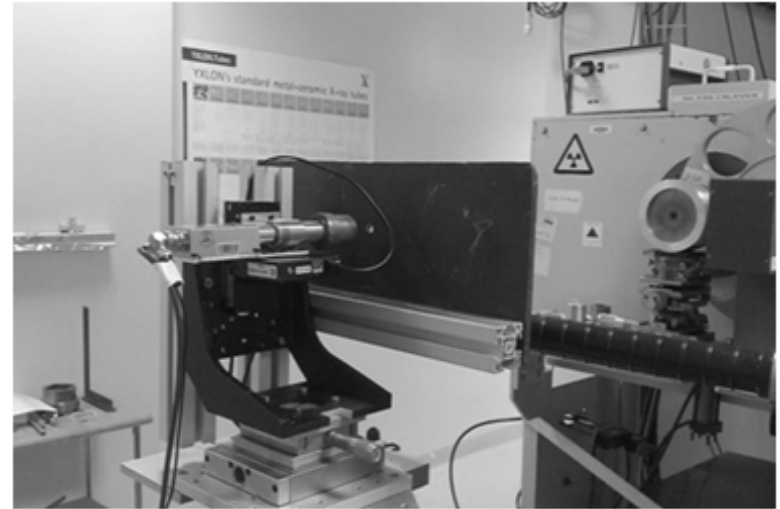

Fig. 3. - Banc avec un détecteur $\mathrm{CdTe}$ face au tube à rayons $\mathrm{X}$ Seifert $320 \mathrm{kV}$.

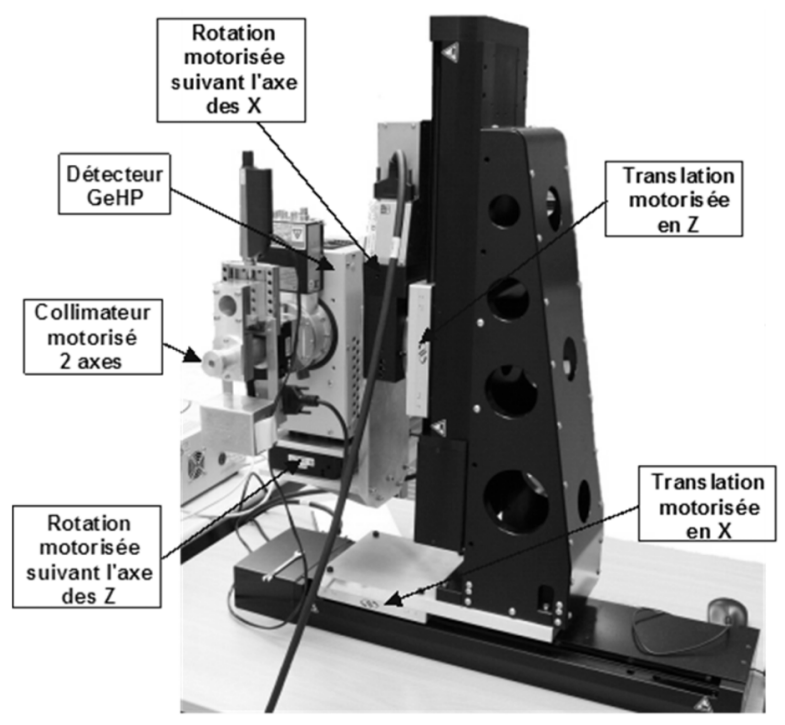

Fig. 4. - Banc de positionnement d'un détecteur lourd.

un encombrement réduit et est adapté aux détecteurs de faible poids (Si-PIN et CdTe). Le second est prévu pour le déplacement de détecteurs plus lourds comme le GeHP.

Le premier banc de positionnement comporte deux axes de translation réglables manuellement (précision de l'ordre de $0,01 \mathrm{~mm}$ ) et deux rotations motorisées (avec un pas de l'ordre de $0,01^{\circ}$ ), ce qui est amplement suffisant pour les alignements recherchés. Tout ce matériel compose un ensemble compact permettant de réaliser aisément des mesures sur site dans les milieux médicaux ou industriels. Un exemple de mise en place de ce banc dans l'axe du faisceau du tube à rayons X Seifert 320 kV est montré sur la photographie de la figure 3.

Le deuxième banc de positionnement est équipé de deux plateformes de translation motorisées (précision de l'ordre de $1 \mu \mathrm{m}$ ) permettant d'explorer un plan de $30 \mathrm{~cm}^{2}$. Il est également doté de deux plateaux tournants motorisés avec un pas inférieur à $0,01^{\circ}$ (fig. 4).

Un exemple de mise en place de ce banc dans l'axe du faisceau du mammographe est montré sur la figure 5.

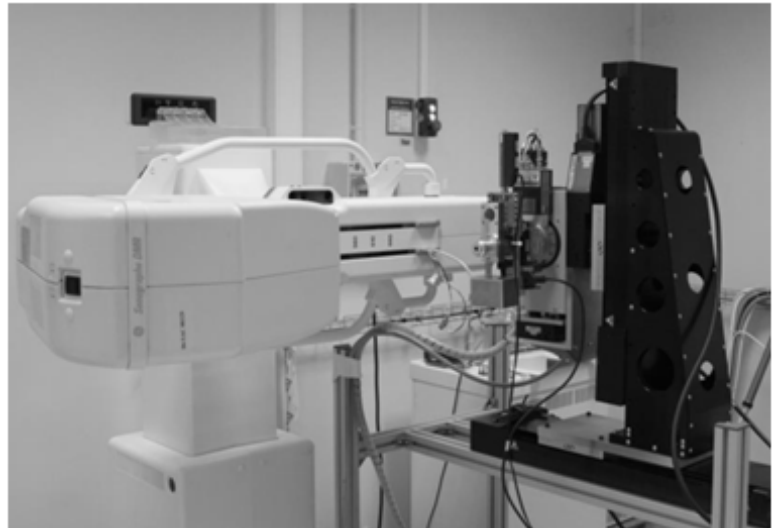

Fig. 5. - Banc avec un détecteur GeHP face au mammographe.

Sur ces deux bancs, les deux mouvements de rotation sont utilisés pour aligner le couple détecteur/collimateur sur l'axe du faisceau. Chaque banc est piloté via un logiciel spécifique réalisé au LNE-LNHB. Grâce aux fonctions qui ont été développées dans ces logiciels, il est possible de mesurer et d'enregistrer les spectres d'énergie du faisceau pour chaque position du détecteur face au faisceau.

Les distances adoptées pour la mesure des spectres en énergie sur chaque installation sont de $50 \mathrm{~cm}$ pour le mammographe et le tube à rayons X Gulmay 160 kV, et de $120 \mathrm{~cm}$ pour le tube Seifert $320 \mathrm{kV}$. Elles correspondent à la distance séparant le foyer du tube $\mathrm{X}$ à la fenêtre d'entrée des détecteurs.

\section{Corrections sur les spectres mesurés}

En fonction des gammes d'énergies émises par les générateurs de rayons $\mathrm{X}$, les spectres mesurés présentent des déformations plus ou moins importantes par rapport aux distributions énergétiques réelles émise par la source. Ces erreurs de mesure sont liées aux processus d'interaction des photons dans le détecteur. Il est donc important de pouvoir corriger les mesures de ces artefacts de détection. Des algorithmes mathématiques intégrant cinq types de corrections ont donc été développés.

\subsection{Correction due aux empilements électroniques}

Lorsque le taux de comptage est important, la probabilité de voir se superposer plusieurs signaux électroniques, issus de l'interaction de photons dans le détecteur n'est plus négligeable. Ce phénomène est dû aux empilements électroniques. Il se traduit par un accroissement du fond du spectre dans la région des fortes énergies. Afin de corriger ces déformations, un algorithme de calcul de la probabilité d'empilement a été développé au LNELNHB. La méthode de calcul utilisée permet d'obtenir le spectre d'empilements correspondant par la superposition des énergies d'un spectre sur elles-mêmes. Le calcul est basé sur une succession de calculs itératifs (méthode de stripping), énergies par énergies, en partant des basses énergies vers les plus hautes. 


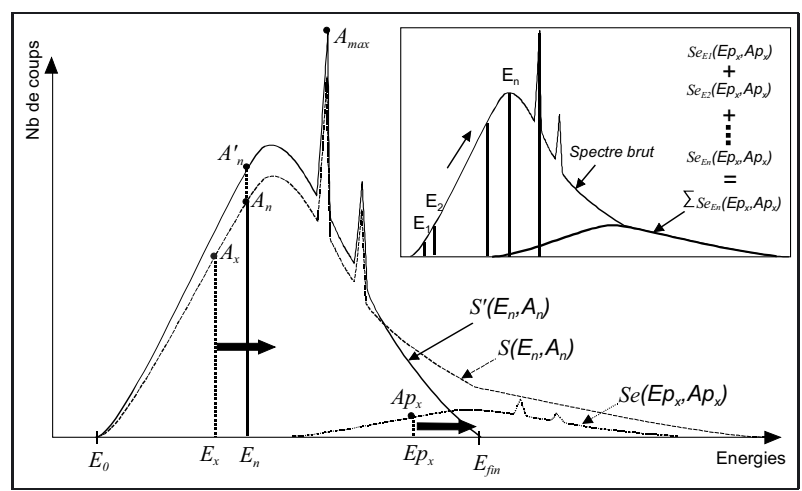

Fig. 6. - Paramètres utilisés pour calculer le spectre d'empilement.

Considérons un canal $n$, l'énergie $E_{n}$ correspondante, et son amplitude $A_{n}$ tiré d'un spectre mesuré $S\left(E_{n}, A_{n}\right)$ (fig. 6).

L'empilement de l'énergie $E_{n}$ sur toutes les autres énergies $E_{x}$ (d'amplitude $A_{x}$ ) appartenant au spectre $S$ correspond à un spectre d'empilement $\operatorname{SeE}_{n}\left(E p_{x}, A p_{x}\right)$ associé à $E_{n}$. $\operatorname{Se} E_{n}\left(E p_{x}, A p_{x}\right)$ est caractérisé par l'équation (1) avec $x$ étant un nombre entier compris entre 0 et le canal maximal du spectre (i.e. : l'énergie maximale du spectre).

$$
\operatorname{SeE}_{n}\left(E p_{x}, A p_{x}\right) \Rightarrow \quad \mid \begin{aligned}
& E p_{x}=E_{n}+E_{x} \\
& A p_{x}=\frac{A_{n}}{A_{\max }} \cdot \operatorname{coef} \cdot A_{x}
\end{aligned}
$$

Le paramètre coef est une constante permettant d'ajuster l'amplitude du spectre d'empilement. Quand le calcul de $\operatorname{SeE}_{n}\left(E p_{x}, A p_{x}\right)$ est réalisé, la nouvelle amplitude de $A_{n}^{\prime}$ peut être obtenue en utilisant l'expression (2).

$$
A_{n}^{\prime}=A_{n}+\sum_{x=0}^{x=\text { end }} A p_{x}
$$

Cette expression est utilisée pour corriger l'amplitude $A_{n}$ de la perte des signaux qui créent le spectre d'empilement $S e E_{n}$. À partir de la connaissance du spectre d'empilement $\operatorname{SeE}_{n}\left(E p_{x}, A p_{x}\right)$, le spectre corrigé $S^{\prime}\left(E_{n}, A_{n}^{\prime}\right)$ est obtenu en utilisant l'équation (3).

$$
S^{\prime}\left(E_{n}, A_{n}^{\prime}\right)=S\left(E_{n}, A_{n}\right)-\operatorname{SeE}_{n}\left(E p_{x}, A p_{x}\right)
$$

À ce stade, $n$ est incrémenté à $n+1$ et la procédure de calcul du nouveau spectre d'empilement $\operatorname{SeE}(n+1)$ est répétée pour le canal suivant. Le paramètre coef est obtenu en réalisant des essais successifs de traitement des spectres. L'amplitude du fond du spectre dans la région des hautes énergies permet de déterminer la meilleure valeur de ce paramètre. Elle doit être proche de zéro.

La figure 7 montre un exemple de correction des empilements sur un spectre mesuré avec le détecteur CdTe sur le tube Seifert réglé à $100 \mathrm{kV}$. Après traitement du spectre, le fond lié aux phénomènes d'empilement visible

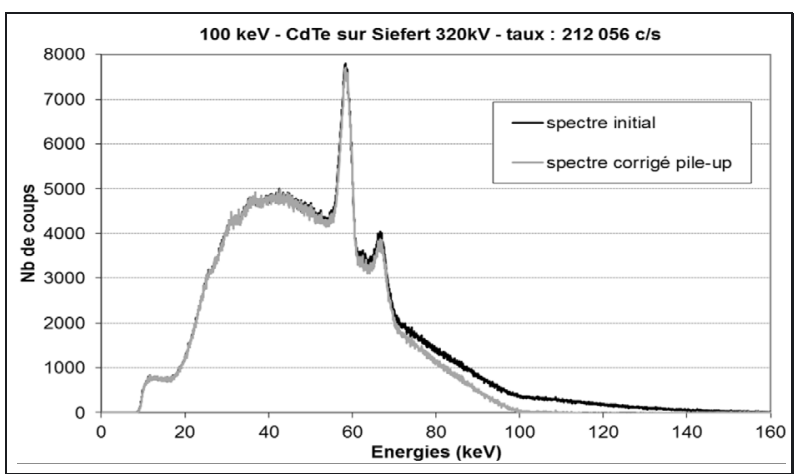

Fig. 7. - Exemple de la correction due aux empilements électroniques.

entre $70 \mathrm{keV}$ et $140 \mathrm{keV}$ a disparu et la valeur du seuil à $100 \mathrm{kV}$ est bien retrouvée malgré un taux de comptage supérieur à $200000 \mathrm{~s}^{-1}$.

\subsection{Correction due à la forme de la réponse spectrale des détecteurs}

Lors de l'interaction d'un photon dans un détecteur, l'énergie transportée par ce photon peut ne pas être absorbée dans son intégralité. Une fraction peut être perdue suite à des phénomènes de pertes d'électrons secondaires, de diffusion Compton, d'échappements de photons de fluorescence ou de recombinaisons de porteurs de charge. Le spectre alors obtenu présente des déformations situées à gauche de la raie principale (pic d'absorption totale avec effet photo-électrique majoritaire). Il est alors évident que le spectre mesuré avec un détecteur à semiconducteur ne représente pas le spectre réellement émis par une source de rayonnement.

La difficulté pour corriger ces artéfacts vient du fait qu'ils évoluent de manière importante en fonction de l'énergie. Pour réaliser les algorithmes de correction, il est indispensable de caractériser l'évolution des réponses spectrales des détecteurs dans une large plage d'énergies à l'aide de rayonnements mono-énergétiques. De ce fait, une campagne de caractérisation des deux détecteurs GeHP et CdTe a été menée dans la gamme d'énergies comprises entre $26,5 \mathrm{keV}$ et $250 \mathrm{keV}$ sur la ligne de lumière ID17 de l'ESRF à Grenoble. Seize réponses spectrales à un rayonnement mono-énergétique ont ainsi été mesurées sur chacun des détecteurs, avec un pas de $10 \mathrm{keV}$ entre $30 \mathrm{keV}$ et $150 \mathrm{keV}$.

Les figures 8 et 9 montrent des exemples des réponses spectrales des détecteurs GeHP et CdTe obtenues pour les trois énergies $30 \mathrm{keV}, 120 \mathrm{keV}$ et $250 \mathrm{keV}$.

À partir de la connaissance de ces réponses spectrales, des modèles mathématiques permettant de calculer ces réponses ont été élaborés pour les deux détecteurs du laboratoire. La figure 10 montre le modèle général adopté pour calculer le fond d'un spectre quel que soit le détecteur. 


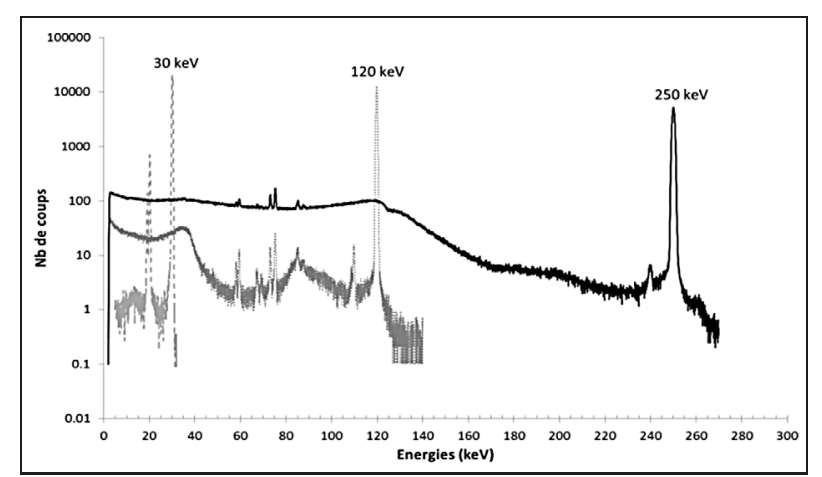

Fig. 8. - Spectres de réponses spectrales du détecteur GeHP aux énergies $30 \mathrm{keV}, 120 \mathrm{keV}$ et $250 \mathrm{keV}$.

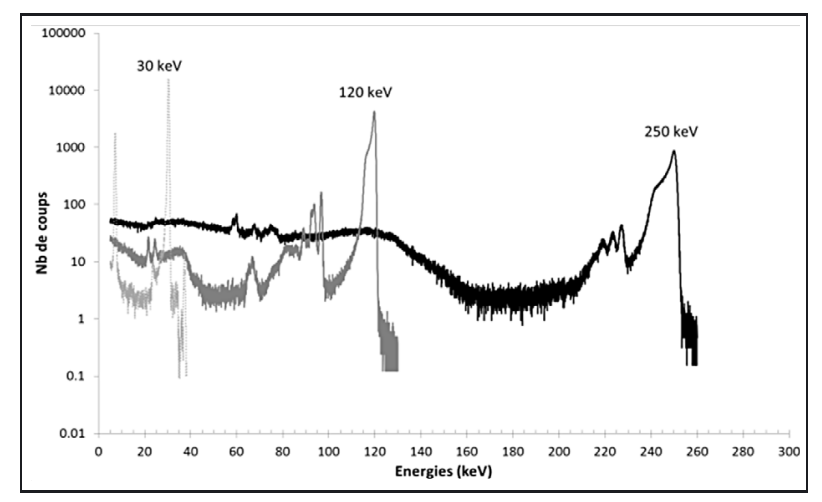

Fig. 9. - Spectres de réponses spectrales du détecteur CdTe aux énergies $30 \mathrm{keV}, 120 \mathrm{keV}$ et $250 \mathrm{keV}$.

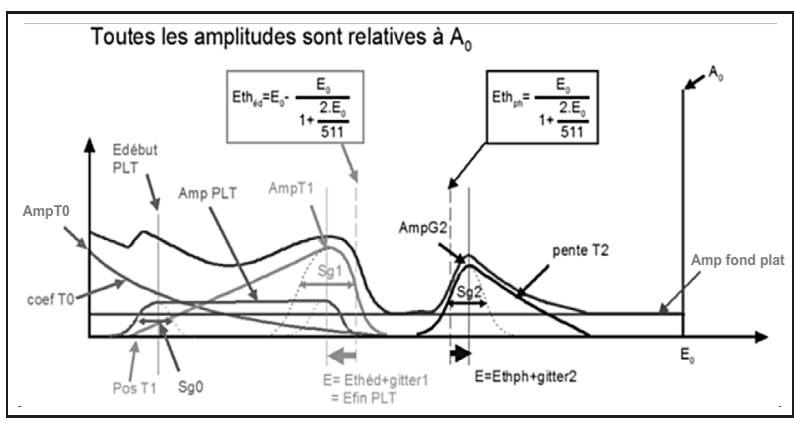

Fig. 10. - Modèles mathématiques (fonctions élémentaires et paramètres) décrivant le fond spectral.

Il est constitué de 9 fonctions mathématiques décrites par 14 paramètres. La connaissance de ces paramètres en fonction de l'énergie permet de calculer le «fond élémentaire » généré par une énergie comprise entre une dizaine de kiloélectronvolt et $250 \mathrm{keV}$.

Afin de vérifier la pertinence des calculs des fonds élémentaires des spectres à partir des fonctions mathématiques et de leurs coefficients associés, des comparaisons entre les fonds calculés et les fonds réels ont été réalisés sur l'ensemble des faisceaux mesurés à l'ESRF avec nos deux détecteurs GeHP et CdTe. Les figures 11 et 12 montrent quelques exemples de comparaison sur les spectres obtenus avec un rayonnement monoénergétique à $140 \mathrm{keV}$ mesurés avec les deux détecteurs. La bonne

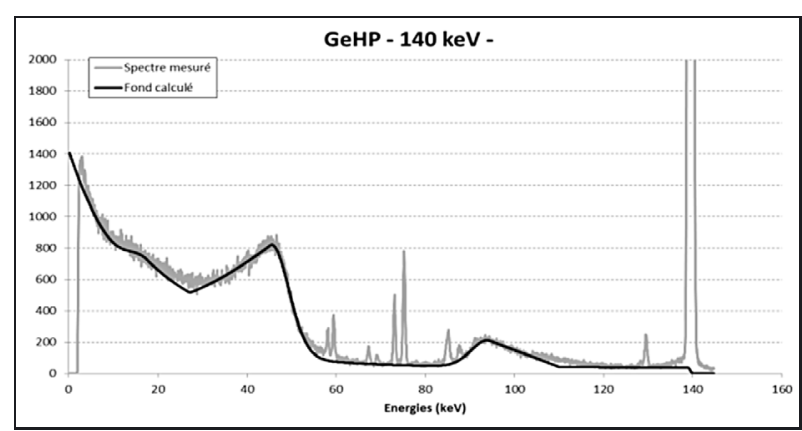

Fig. 11. - Comparaison du fond calculé et du spectre mesuré à $140 \mathrm{keV}$ sur le détecteur GeHP.

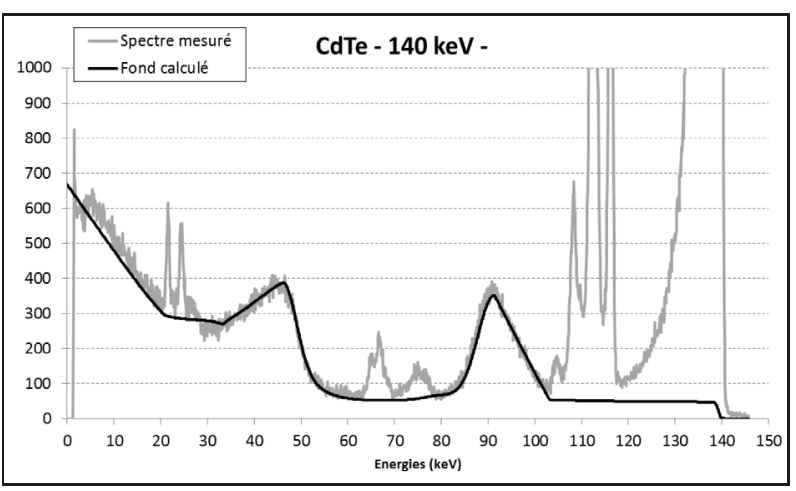

Fig. 12. - Comparaison du fond calculé et du spectre mesuré à $140 \mathrm{keV}$ sur le détecteur CdTe.

concordance entre les calculs et les fonds réels y est bien visible.

L'étape suivante consiste à réaliser une accumulation de ces spectres élémentaires afin que le fond global obtenu coïncide avec celui d'un spectre continu issu d'un générateur de rayons $X$. Une fois de plus, la méthode de stripping, déjà utilisée précédemment pour les corrections d'empilements, a été utilisée.

Le calcul débute en partant des énergies supérieures du spectre. À l'énergie $E_{n}$, d'amplitude $A_{n}$, on calcule les différents paramètres décrivant le spectre de fond élémentaire $S_{f} E_{n}$ généré par $E_{n}$ en utilisant les fonctions obtenues à partir de l'étude des réponses spectrales. Le calcul du spectre $S_{f} E_{n}$ est ensuite réalisé, puis il est soustrait au spectre $S_{\text {continu }}$ initialement mesuré. Parallèlement à cela, le calcul de l'intégrale du spectre $S_{f} E_{n}$ est réalisé et la valeur obtenu $\Sigma S_{f} E_{n}$ est ajoutée à l'amplitude $A_{n}$ de l'énergie $E_{n}$. A ce stade, cette procédure de calcul est réitérée pour l'énergie $E_{n-1}$. La figure 13 illustre la méthode de calcul développée.

Un exemple de comparaison entre un fond calculé par notre méthode et le spectre continu du faisceau N150 mesuré avec le détecteur GeHP est montré sur la figure 14.

\subsection{Correction de la traîne des pics présente sur les spectres du détecteur CdTe}

Les raies visibles sur les spectres mesurés avec le détecteur $\mathrm{CdTe}$ présentent une traîne à gauche plus ou moins importante en fonction de l'énergie. Pour corriger 


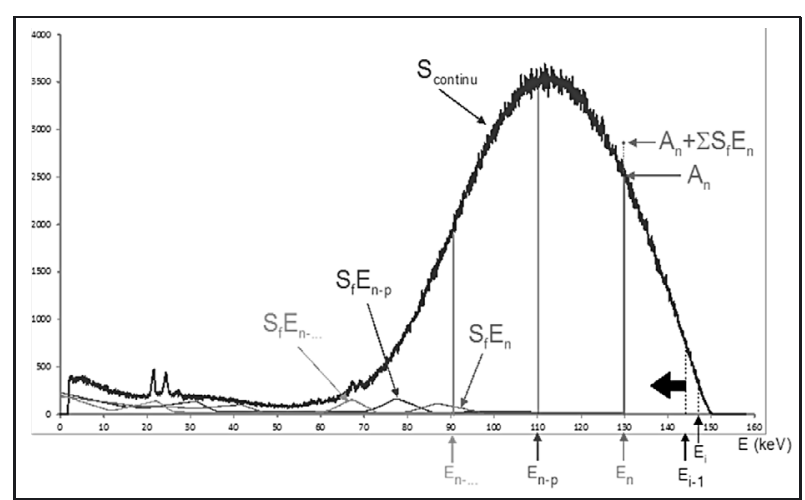

Fig. 13. - Calculs des spectres de fond élémentaires constituant un spectre continu d'un tube $\mathrm{X}$ réglé à $150 \mathrm{kV}$.

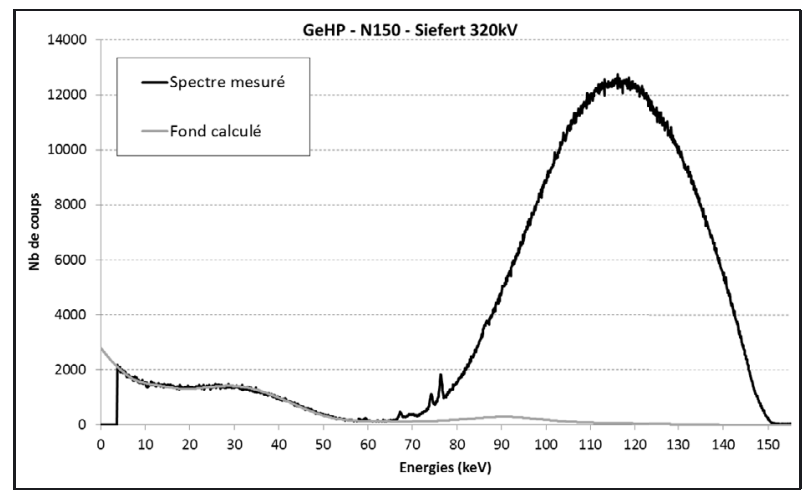

Fig. 14. - Comparaison entre le fond calculé et le spectre continu du spectre N150 mesuré avec le détecteur GeHP.

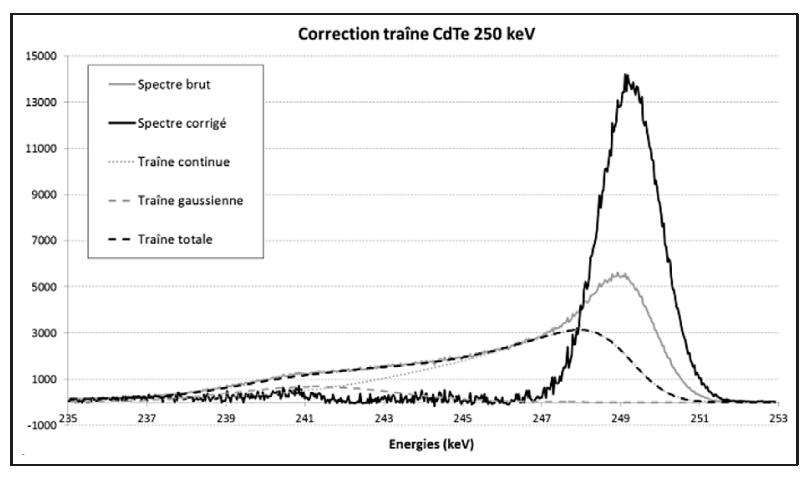

Fig. 15. - Calcul puis soustraction de la traîne de la réponse du détecteur CdTe à $250 \mathrm{keV}$.

ce phénomène, l'étude de l'évolution de cette traîne en fonction de l'énergie a été réalisée à partir des réponses spectrales mesurées à l'aide de l'ESRF.

Deux fonctions mathématiques, permettant de décrire la forme de la traîne quelle que soit l'énergie l'ayant générée, ont été définies. La première fonction correspond à une fonction polynômiale de degré deux, la seconde à une gaussienne simple. La connaissance des paramètres de ces fonctions mathématiques en fonction de l'énergie permet de calculer la forme de la traîne quelle que soit l'énergie considérée.

La figure 15 montre un exemple de calcul de la traîne pour une énergie mono-énergétique incidente de $250 \mathrm{keV}$.
Arrivé à ce stade, la méthode choisie pour étendre le calcul à un spectre continu correspond toujours à la méthode dite de «stripping». Le processus de traitement débute par les énergies les plus hautes du spectre. Pour chaque énergie $E_{n}$ d'amplitude $A_{n}$, le calcul de la traîne est effectué. Une fois le calcul réalisé, le spectre de traîne obtenu, issu de l'énergie $E_{n}$, est soustrait de l'ensemble du spectre continu initial. Parallèlement, le calcul de l'intégrale du spectre de la traîne est effectué puis la valeur obtenue est ajouté à l' amplitude $A_{n}$. À ce stade, on réitère le calcul pour l'énergie $E_{n-1}$.

\subsection{Correction des phénomènes d'échappement}

L'interaction des photons dans le détecteur peut conduire à la création de photons de fluorescence caractéristiques du milieu d'interaction. Si ces photons s'échappent du détecteur, seule une partie de l'énergie incidente est détectée. Cela conduit à l'apparition sur les spectres mesurés de pics dits «d'échappement» dans le cas de rayonnements incidents mono-énergétiques. Dans le cas d'un rayonnement $\mathrm{X}$ continu, la signature des échappements sur le signal mesuré correspond à un autre spectre continu à plus basse énergie. Afin de corriger ce phénomène, il faut connaître son évolution en fonction de l'énergie et du type de détecteur considéré. Une fois de plus, la connaissance des réponses spectrales des détecteurs à un rayonnement mono-énergétique permet de connaître cette évolution. Les rapports $\left(A_{\text {escx }} / A_{n}\right)$ entre les surfaces des pics d'échappement et celle du pic principal ont été mesurés dans la gamme $30 \mathrm{keV}$ à $250 \mathrm{keV}$.

La méthode de correction des échappements repose, une fois de plus, sur la technique de stripping en débutant les calculs par l'énergie maximale $E_{n}$ d'amplitude $A_{n}$. La connaissance des rapports $\left(A_{\text {escx }} / A_{n}\right)$ permet de calculer l'amplitude $A_{e s c x}$ de chaque pic d'échappement escx à partir de l'amplitude $A_{n}$ ( $x$ correspond au numéro de l'échappement: 1 ou 2 pour le germanium, 1, 2, 3 ou 4 pour le CdTe). La position $E_{\text {esc }}$ des pics d'échappement est, quant à elle, parfaitement connue car elle dépend du photon X s'étant échappé : par exemple pour le détecteur $\mathrm{GeHP}, E_{\text {esc } 1}=E_{n}-9,88 \mathrm{keV}$ et $E_{e s c 2}=E_{n}-10,98 \mathrm{keV}$. La quantité $A_{\text {escx }}$ est soustraite de l'amplitude $A_{s p c, e s c x}$ du spectre située à l'énergie $E_{\text {escx }}$ puis cette quantité est ajoutée à l'amplitude $A_{n}$ de l'énergie $E_{n}$ pour compenser la perte du photon s'étant échappé. Pour une énergie $E_{n}$, ce calcul est effectué pour chaque échappement, soit deux fois pour le détecteur germanium (échappement $\mathrm{Xk}_{\alpha}$ et $\mathrm{Xk}_{\beta} \mathrm{du} \mathrm{Ge}$ ) et quatre fois pour le détecteur CdTe (échappements $\mathrm{Xk}_{\alpha}$ et $\mathrm{Xk}_{\beta}$ du $\mathrm{Cd}$ et du Te). Une fois les corrections effectuées à l'énergie $E_{n}$, le processus de calcul est réitéré pour l'énergie précédente à $E_{n-1}$. Chaque calcul est arrêté lorsque l'énergie $E_{n}$ atteint le seuil d'énergie de liaison du matériau considéré $(11,103 \mathrm{keV}$ pour le $\mathrm{Ge}, 26,711 \mathrm{keV}$ pour le $\mathrm{Cd}$ et $31,814 \mathrm{keV}$ pour le Te). La figure 16 illustre ce processus de calcul appliqué au détecteur GeHP sur un spectre à $20 \mathrm{kV}$ de la série N.

La figure 17 montre le même traitement effectué sur un spectre à $40 \mathrm{kV}$ mesuré avec le détecteur CdTe. 


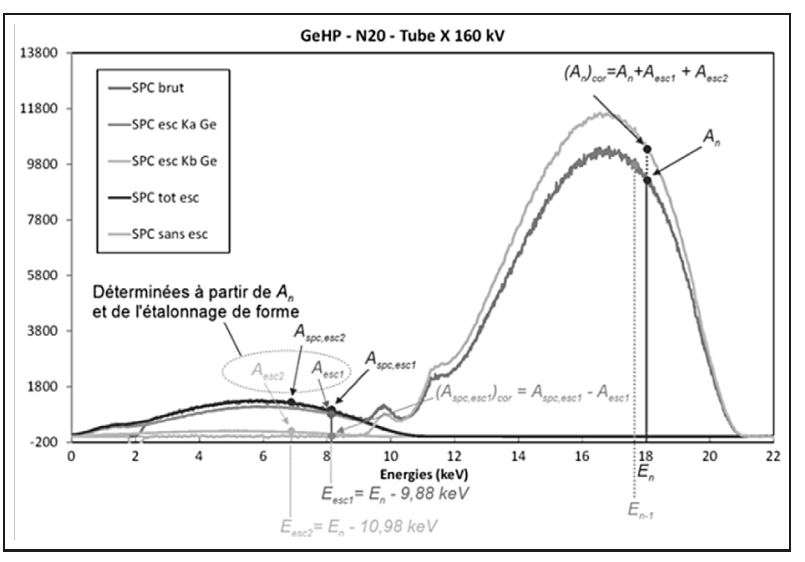

Fig. 16. - Processus de correction des échappements pour le détecteur GeHP.

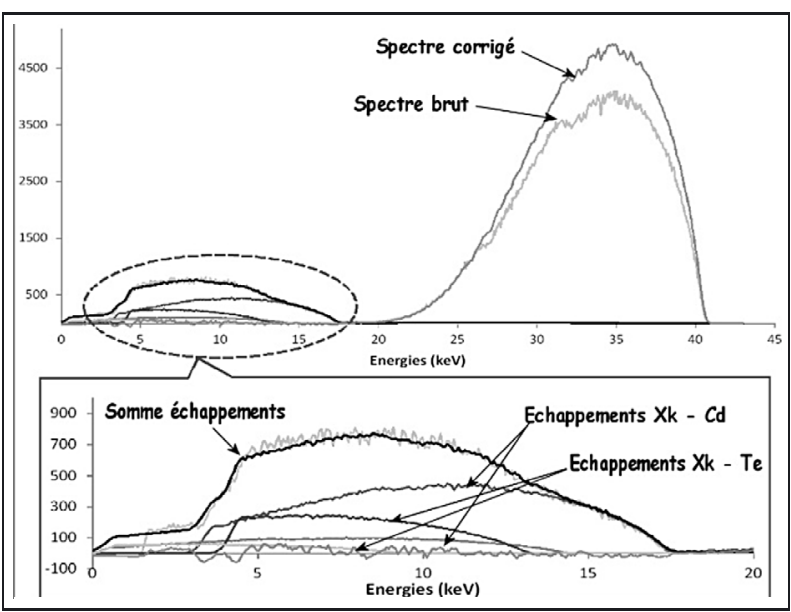

Fig. 17. - Processus de correction des échappements pour le détecteur CdTe.

Ces résultats démontrent que les algorithmes développés pour les deux détecteurs du laboratoire permettent de calculer avec une bonne précision les différents spectres d'échappement, de les soustraire du spectre initial et de compenser les pertes de détection occasionnées par ce phénomène.

\subsection{Correction des rendements de détection}

La dernière correction à apporter aux spectres mesurés correspond à la correction due au rendement de détection. Selon le détecteur, la détermination des courbes de rendement des détecteurs a été réalisée soit par calcul soit par mesure. Pour les détecteurs Si-PIN et GeHP, les valeurs de rendement ont été mesurées à l'aide de trois sources étalons correspondant aux radionucléides suivants : ${ }^{241} \mathrm{Am},{ }^{133} \mathrm{Ba}$ et ${ }^{152} \mathrm{Eu}$. Ils permettent de couvrir une gamme d'énergies comprises entre $12 \mathrm{keV}$ et $380 \mathrm{keV}$. Pour les valeurs entre $5 \mathrm{keV}$ et $17 \mathrm{keV}$, la source de rayonnement SOLEX [4] du LNE-LNHB/ LMA a été utilisée. Pour le détecteur CdTe, la courbe de rendement utilisée correspond à celle fournie par le constructeur. Il s'agit d'une courbe calculée à partir des épaisseurs des différents éléments du détecteur (fenêtre d'entrée et cristal CdTe).

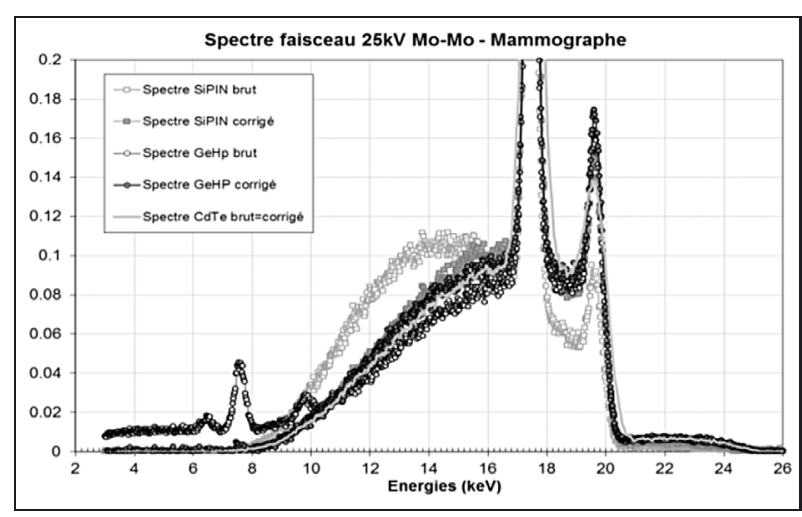

Fig. 18. - Spectres en énergie, bruts et corrigés, pour le faisceau « $25 \mathrm{kV}$ (Mo-Mo)».

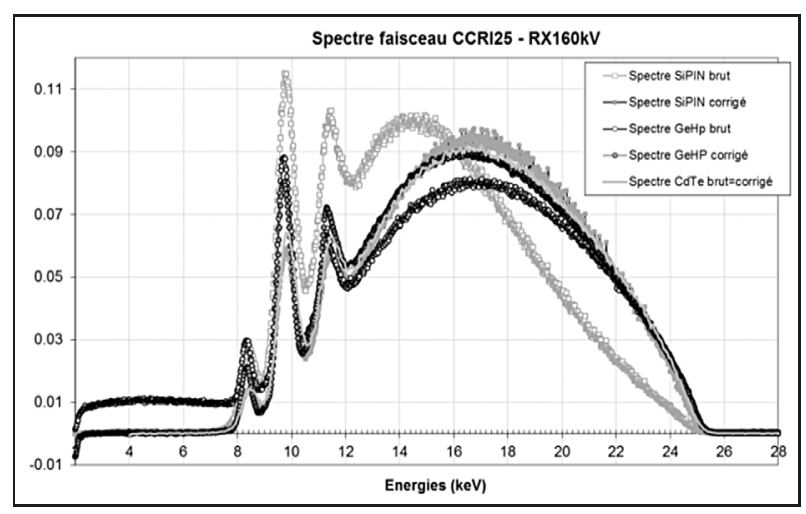

Fig. 19. - Spectres en énergie, bruts et corrigés, pour le faisceau « CCRI $25 »$.

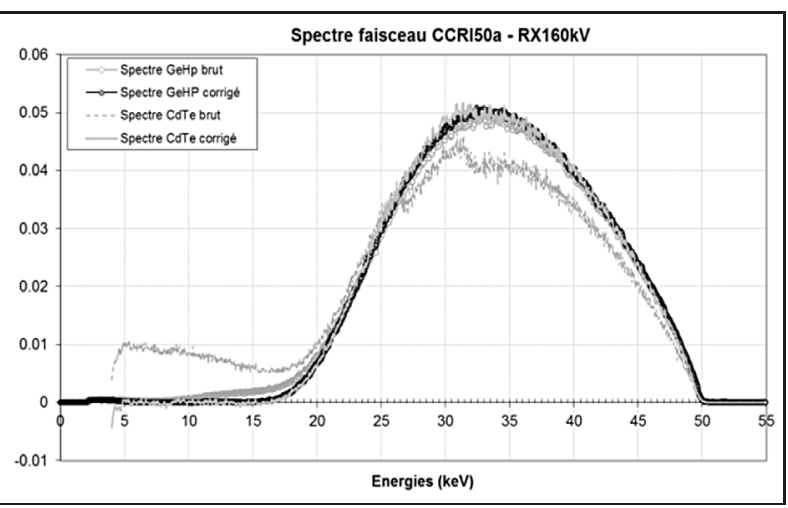

Fig. 20. - Spectres en énergie, bruts et corrigés, pour le faisceau « CCRI50a ».

\section{Résultats de mesure de spectres en énergie de faisceaux de rayons $X$}

Nous ne présentons pas ici l'intégralité des résultats obtenus sur les 28 faisceaux mesurés. L'ensemble des résultats est consultable sur le site internet du LNELNHB [5]. Quelques exemples de spectres en énergie des faisceaux mesurés sur les générateurs de rayons $\mathrm{X}$ du laboratoire sont montrés sur les figures 18-22. Ces spectres d'énergie ont tous été corrigés des artéfacts de détection liés aux empilements électroniques, aux réponses spectrales de chaque détecteur, aux échappements $(\mathrm{Ge}$, $\mathrm{Cd}, \mathrm{Te}$ ) et aux rendements des détecteurs. 


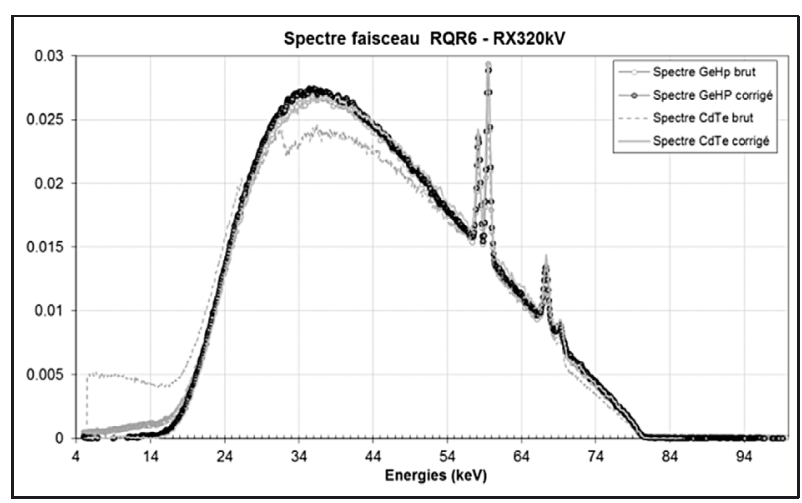

Fig. 21. - Spectres d'énergie, bruts et corrigés, pour le faisceau «RQR6».

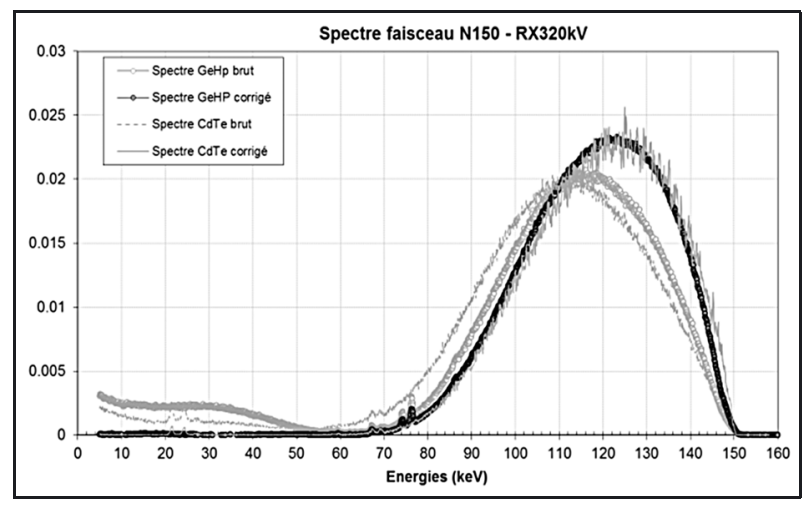

Fig. 22. - Spectres en énergie, bruts et corrigés, pour le faisceau « N150».

Les résultats obtenus sur les faisceaux « $25 \mathrm{kV}$ Mo-Mo » (fig. 18) et «CCRI25 » (fig. 19) permettent de constater que la correction des échappements du détecteur GeHP fonctionne parfaitement. Après correction, les spectres d'énergie mesurés avec ce détecteur se superposent bien à ceux obtenus avec le détecteur CdTe qui, lui, ne présente pas d'échappements dans la gamme d'énergies couverte par ces deux faisceaux.

La même remarque peut s'appliquer au détecteur CdTe avec le spectre en énergie du faisceau «CCRI50a » (fig. 20) où l'inverse se produit : les échappements sont importants dans le détecteur CdTe, et peu intenses dans le détecteur GeHP. La bonne superposition des deux spectres corrigés prouve le bon fonctionnement des corrections mises en œuvre. Il en va de même pour le faisceau « RQR6» (fig. 21).

Concernant la correction du fond résiduel des spectres, les résultats obtenus à plus «hautes énergies » montrent une bonne réduction des fonds avec les deux détecteurs utilisés comme en témoignent les résultats obtenus sur le faisceau «N150» (fig. 22).

\section{Comparaison des spectres en énergie mesurés et calculés}

Il existe plusieurs logiciels commercialisés permettant de calculer le spectre en énergie de l'émission X

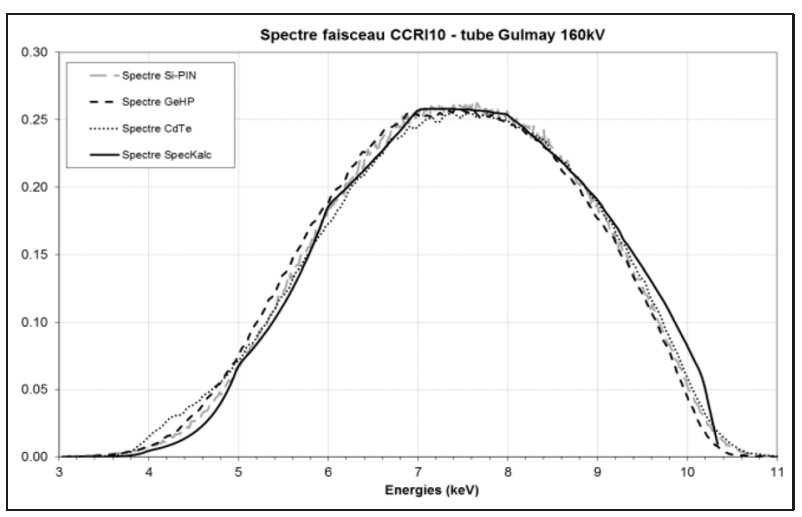

Fig. 23. - Spectres en énergie, mesurés et calculés, pour le faisceau «CCRI10».

d'un tube à rayons X. Généralement, pour réaliser ce type de calcul, ces logiciels utilisent les données du constructeur décrivant la structure interne du tube. Il est évident que la qualité des résultats obtenus dépend de la complexité du modèle de calcul développé ainsi que de la prise en compte de l'ensemble de la structure interne de l'appareil. Les logiciels ne fournissent donc pas tous des résultats de même qualité. Les phénomènes d'interaction des électrons dans l'anode, conduisant à l'émission des rayons $\mathrm{X}$, sont nombreux et complexes. Ils dépendent d'un grand nombre de paramètres dont certains sont mal connus (vieillissement de l'anode par exemple). Il est donc important de pouvoir évaluer la fiabilité de chaque logiciel. Nous avons donc comparé les résultats des mesures effectuées au LNHB à ceux fournis par deux logiciels de calcul de spectres en énergie du commerce (XCOMP5r et SpekCalc V1.0). Les données d'entrées utilisées pour les calculs sont relativement simples : haute tension, angle d'anode, distance où l'on souhaite avoir le spectre, filtration (limitée à quelques matériaux tels que $\mathrm{Be}, \mathrm{Al}, \mathrm{Cu}, \mathrm{Sn}, \mathrm{Pb}$, eau...). Le logiciel XCOMP5r ne permet pas de calculer les énergies pour des hautes tensions inférieures à 20 kV et supérieures à 150 kV.

La figure 23 montre les résultats obtenus pour le spectre en énergie du faisceau «CCRI10» délivré par le tube $\mathrm{X}$ « Gulmay $160 \mathrm{kV}$ ». Dans la gamme d'énergies délivrée par ce faisceau, les seules corrections à prendre en compte correspondent aux phénomènes d'empilement et aux rendements de détection de chaque détecteur. Les résultats obtenus sont très comparables compte tenu du domaine d'énergies très bas. Les résultats fournis par le logiciel SpekCalc sont très comparables aux spectres mesurés malgré l'influence de la nature des matériaux et de leurs variations d'épaisseur sur l'absorption du rayonnement dans cette gamme d'énergies.

La figure 24 fournit les résultats pour le faisceau «CCRI25 » issu du tube Gulmay $160 \mathrm{kV}$. Le premier point surprenant concerne l'absence de raies $L$ de fluorescence du tungstène sur le spectre en énergie calculé avec le logiciel SpekCalc. Dans le calcul avec le logiciel XCOMP5r, ces raies sont présentes mais leurs positions semblent peu précises. La raie $L_{\beta}(9,74 \mathrm{keV})$ est placée 


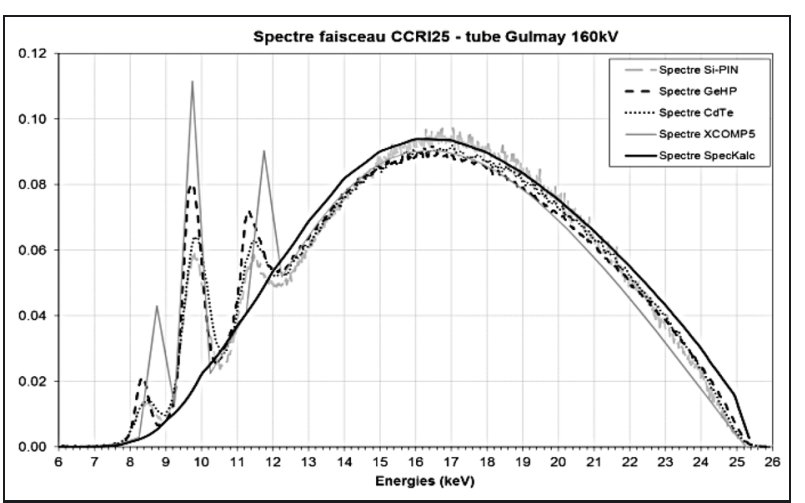

Fig. 24. - Spectres en énergie, mesurés et calculés, pour le faisceau « CCRI25 ».

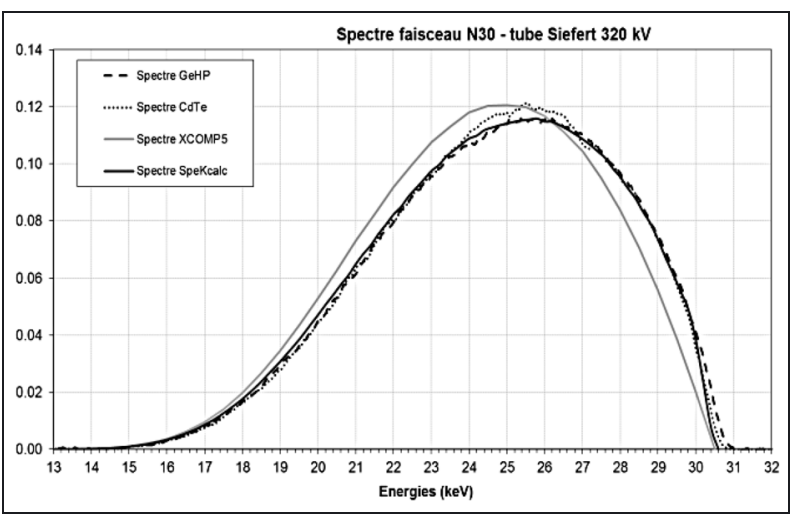

Fig. 25. - Spectres en énergie, mesurés et calculés, pour le faisceau « N30».

à la bonne énergie ce qui n'est pas le cas des raies $L_{\alpha}$ $(8,39 \mathrm{keV})$ et $L_{\gamma}(11,32 \mathrm{keV})$.

La figure 25 montre les spectres en énergie mesurés et calculés pour le faisceau «N30» du tube « Seifert $320 \mathrm{kV} »$.

La correspondance entre les spectres en énergie mesurés et celui calculé avec le logiciel «SpekCalc V1.0» est très bonne. Un décalage vers les basses énergies du spectre fourni par le logiciel «XCOMP5r» est visible tout en gardant des énergies de seuil (début et fin du spectre) similaires à celles observées sur les autres spectres.

Les résultats pour le faisceau «CCRI50a » sont montrés sur la figure 26. Avec une énergie moyenne supérieure à $30 \mathrm{keV}$, le détecteur Si-PIN n'a pas été utilisé pour la mesure de ce faisceau compte tenu des corrections de rendement trop importantes à appliquer. La filtration relativement importante de ce faisceau permet d'absorber les raies L de fluorescence du tungstène. De ce fait, les calculs effectués avec le logiciel SpekCalc ne présentent plus les biais observés précédemment. Les résultats sont parfaitement homogènes, toutes méthodes et détecteurs confondus.

La comparaison entre les mesures et les calculs pour le faisceau «RQR6» est montrée sur la figure 27. L'une des particularités des faisceaux de la série $\mathrm{RQR}$ concerne

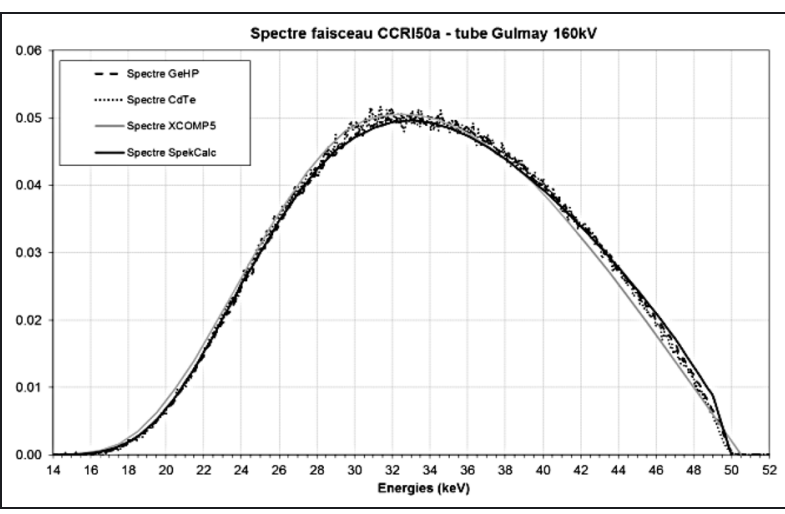

Fig. 26. - Spectres en énergie, mesurés et calculés, pour le faisceau «CCRI50a».

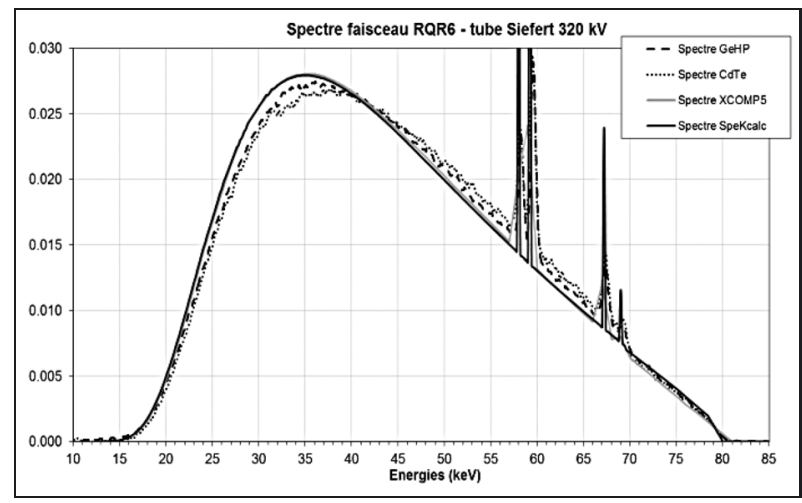

Fig. 27. - Spectres en énergie, mesurés et calculés, pour le faisceau «RQR6».

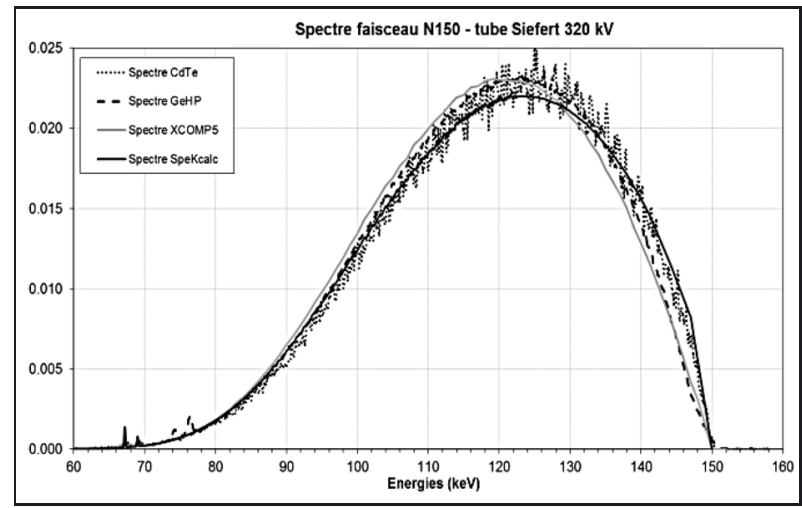

Fig. 28. - Spectres en énergie, mesurés et calculés, pour le faisceau « N150».

leur faible filtration. Cela se traduit par une distribution spectrale en énergie très large débutant dans les basses énergies. Les spectres mesurés sont très cohérents. Concernant les spectres calculés, les résultats sont très similaires pour les deux logiciels. Ils présentent un écart par rapport aux spectres mesurés dans la gamme de $25 \mathrm{keV}$ à $45 \mathrm{keV}$, ce qui se traduit par une énergie moyenne des spectres légèrement décalée vers les basses énergies. Par contre l'énergie du début du spectre $(15 \mathrm{keV})$ coïncide bien avec ce qui est mesuré avec les détecteurs du LNHB. 
Les résultats des calculs réalisés pour le faisceau « N150 » sont représentés sur la figure 28. Il existe un écart entre les formes des spectres calculés, ce qui conduit à une différence d'énergie moyenne entre ces deux spectres d'environ $1 \%$. Malgré cela, les résultats obtenus sont compatibles avec les spectres en énergie mesurés.

A l'issue de l'observation des résultats obtenus sur l'ensemble des 28 faisceaux mesurés et calculés, on constate que pour les faisceaux ne comportant pas les raies de fluorescence $\mathrm{X}_{\mathrm{L}}$ du tungstène, le logiciel « SpekCalc V1.0» semble fournir des spectres en énergie relativement fiables dans toute la gamme de tensions explorée (jusqu'à 150 kV). Concernant le logiciel «XCOMP5r», son avantage est de pouvoir calculer les raies de fluorescence du tungstène même si leurs énergies sont mal placées. Par contre, les résultats qu'il fournit pour certains faisceaux en basse énergie montrent un décalage des spectres vers les faibles énergies (faisceaux N20, N30, N40 et N60). Au-delà, les spectres en énergie calculés sont représentatifs des spectres mesurés.

\section{Conclusion}

Durant ces quatre dernières années, le LNELNHB/LMD s'est attaché à développer des méthodes et des moyens pour mesurer les spectres en énergie de l'émission $\mathrm{X}$ des tubes à rayons $\mathrm{X}$ utilisés au laboratoire pour la réalisation des références en kerma dans l'air et leur transfert vers les utilisateurs dans les domaines tant médicaux qu'industriels. Les travaux de recherche menés ont conduit à la réalisation de deux bancs de mesure utilisant trois types de détecteurs à semi-conducteur (Si-PIN, GeHP, CdTe) adaptés à la gamme d'énergies à mesurer. Equipés de collimateurs en tungstène comportant un très faible diamètre d'ouverture, ces bancs permettent, d'une part, d'aligner avec une très grande précision l'ensemble détecteur-collimateur sur l'axe du faisceau et, d'autre part, de réaliser des mesures de spectres sur des faisceaux à forts débits comme ceux délivrés par les mammographes.

Les spectres en énergie mesurés avec les détecteurs à semi-conducteur comportent des déformations spectrales intimement liées aux processus d'interaction des photons dans le détecteur lui-même. Afin d'obtenir les spectres en énergie réellement émis par les tubes à rayons X à partir

Article reçu le 16 décembre 2015, version révisée reçue le 24 novembre 2016. des spectres mesurés, le laboratoire a développé des algorithmes de correction spécifiques à chaque détecteur. Ils permettent de corriger les déformations liées aux empilements électroniques, aux diffusions des photons dans le détecteur, aux défauts de collection des charges (traînes des pics du détecteur $\mathrm{CdTe}$ ), aux échappements des photons et aux rendements de détection. Le développement de ces algorithmes a été possible grâce à un travail important de caractérisation des réponses spectrales des détecteurs pour un rayonnement mono-énergétique, à l'aide de la ligne de lumière « ID17 » de l'ESRF à Grenoble.

Une campagne de mesure des spectres en énergie des faisceaux de trois installations de rayons $\mathrm{X} \mathrm{du}$ LNHB a été entreprise afin de caractériser les faisceaux de référence utilisés par le laboratoire jusqu'à $150 \mathrm{kV}$. Elle concernait les tubes «Gulmay $160 \mathrm{kV}$ », « Seifert $320 \mathrm{kV} »$ et enfin l'installation de mammographie. Un total de 28 faisceaux répondant aux qualités des faisceaux CCRI, RQR et N ont été mesurés avec trois détecteurs SiPIN, GeHP et CdTe. La comparaison croisée des mesures d'un même faisceau avec les différents détecteurs a permis de valider les algorithmes de correction développés. Ces spectres mesurés ont été comparés aux spectres calculés avec les logiciels commercialisés «XCOMP5r» et «SpekCalc V1.0». À l'issue de cette campagne de mesures, il a été constaté que l'ensemble des spectres en énergie mesurés puis corrigés de leurs défauts de détection étaient en très bon accord les uns avec les autres, quel que soit le faisceau mesuré.

\section{Références}

[1] Norme ISO 4037-1:1996, « Rayonnements X et gamma de référence pour l'étalonnage des dosimètres et des débitmètres, et pour la détermination de leur réponse en fonction de l'énergie des photons - Partie 1: Caractéristiques des rayonnements et méthodes de production ».

[2] Nowotny, XCOMP5, Program for calculating diagnostic X-ray spectra., Roefo. Fortschr. Geb. Roentgenstr. Nuklearmed., 142, 1985, 685-689.

[3] Poludniowski G., Landry G., DeBlois F., Evans P.M. et VERHAEGEN F., "SpekCalc: a program to calculate photon spectra from tungsten anode x-ray tubes", Phys. Medicine Biol., 54, 19, 2009, N433-N438.

[4] BonNELLE C. et al., "SOLEX: a tunable monochromatic $\mathrm{X}$-ray source in the 1-20 keV energy range for metrology", Nucl. Instrum. Meth. in Phys. Res. A, 516, 594, 2003.

[5] www.nucleide.org 